Case report

\title{
Invasive bilateral breast cancer and high grade serous ovarian cancer with BRCA1-germline mutation and brainstem metastasis under PARP inhibitors
}

\author{
Mihaela Mărioara Zahari ${ }^{1, *}$, Angelica Rita Chiorean², Maria Magdalena Duma ${ }^{2}$, \\ Andrei Ungureanu ${ }^{3}$, Gabriel Kacso ${ }^{3}$
}

1"Prof. Dr. Ion Chiricuță" Oncologic Institute, Cluj-Napoca, Romania; ${ }^{2}$ Medimages Clinic, ClujNapoca, Romania; ${ }^{3}$ Amethyst Clinic, Cluj-Napoca, Romania

\begin{abstract}
For breast cancer patients, BRCA gene mutations are predictive of a good response to chemotherapy, but are hampered by a high risk of bilateral and synchronous or metachronous ovarian cancer. Novel therapies such as PARP-inhibitors have proven effective for BRCA1/2 mutated ovarian cancer. We present the case of a 50-year-old woman, initially diagnosed with bilateral luminal B breast cancer with BRCA1 mutation. She received neoadjuvant chemotherapy, modified radical mastectomy and bilateral adnexectomy, while subsequently identifying a synchronous advanced ovarian cancer, stage FIGO IIIC, followed by adjuvant platinum chemotherapy and external radiotherapy. After a 12 months disease-free interval a brainstem tumor was discovered, for which whole-brain radiotherapy was performed. She received 6 months of PARP-inhibitors through an early access program. With only a partial at the end of treatment, the brainstem tumor was still in progression. Due to evolution of the brain metastasis, second line chemotherapy (taxanes and Bevacizumab) was administered, with complete radiologic response. The particularity of this case resides in the coexistence of a breast and ovarian cancer in the same patient with BRCA1-germline mutation who responded to a new line of therapy - the PARP inhibitors. While being unable to perform a biopsy, we speculate that the brain metastasis in this case was most likely of breast origin.
\end{abstract}

Keywords: brain metastasis; BRCA mutation; breast cancer; ovarian cancer; PARP inhibitors

\section{Introduction}

Breast cancer is the second most common cancer in the world and the first in women. It accounts for more than $25 \%$ of all new diagnosis of malignancy and $15 \%$ of cancer deaths in women [1]. The coexistence of breast and ovarian cancer in the same patient should raise the suspicion of a hereditary factor. BRCA mutation makes ovarian cancers more sensitive to DNA targeting therapy such

Received: June 2019; Accepted after review: September 2019; Published: September 2019.

*Corresponding author: Mihaela Mărioara Zahari, "Prof. Dr. Ion Chiricuță" The Oncology Institute, 34-36 Republicii Str., Cluj-Napoca 400015, Romania.

Email: zahari mihaela2000@yahoo.com as platinum agents [2].

Cerebral metastases have an incidence of $10-30 \%$ for breast cancer and much less for ovarian cancer (3-4\%). Most of these patients $(65.5 \%$ in breast cancer, $68.2 \%$ in ovarian cancer) have a BRCA1 mutation [3].

\section{Case report}

A 50-year-old woman presented in our hospital in September 2016 for a mass in the left breast, self-detected by palpation 3 months before. The menarche installed at 15 years, the last menstruation was in February 2016.

The family oncologic history revealed the presence of ovarian cancer in the case of her 
mother, diagnosed at 63. Local clinical examination identified a fixed mass against the underlying tissues, measuring $10 / 11 \mathrm{~cm}$ in the left breast with an inflammatory aspect of the gland, with skin invasion; in the right breast, at the junction of the internal quadrants, a fixed mass measuring $5 / 6 \mathrm{~cm}$ was also objectified. After performing a mammogram (Figure 1), breast ultrasound and elastography, breast MRI (Figure 2), the diagnosis was bilateral luminal B breast cancer - invasive carcinoma NST, Nottingham II, $E R=70 \%, P R=3 \%$, HER2 $=1+$ (negative), Ki67 $=40 \%$, in the left breast and invasive carcinoma NST, Nottingham II, ER=60\%, PR= 1\%, HER2 $=0$ (negative), Ki67 $=50 \%$, in the right breast.

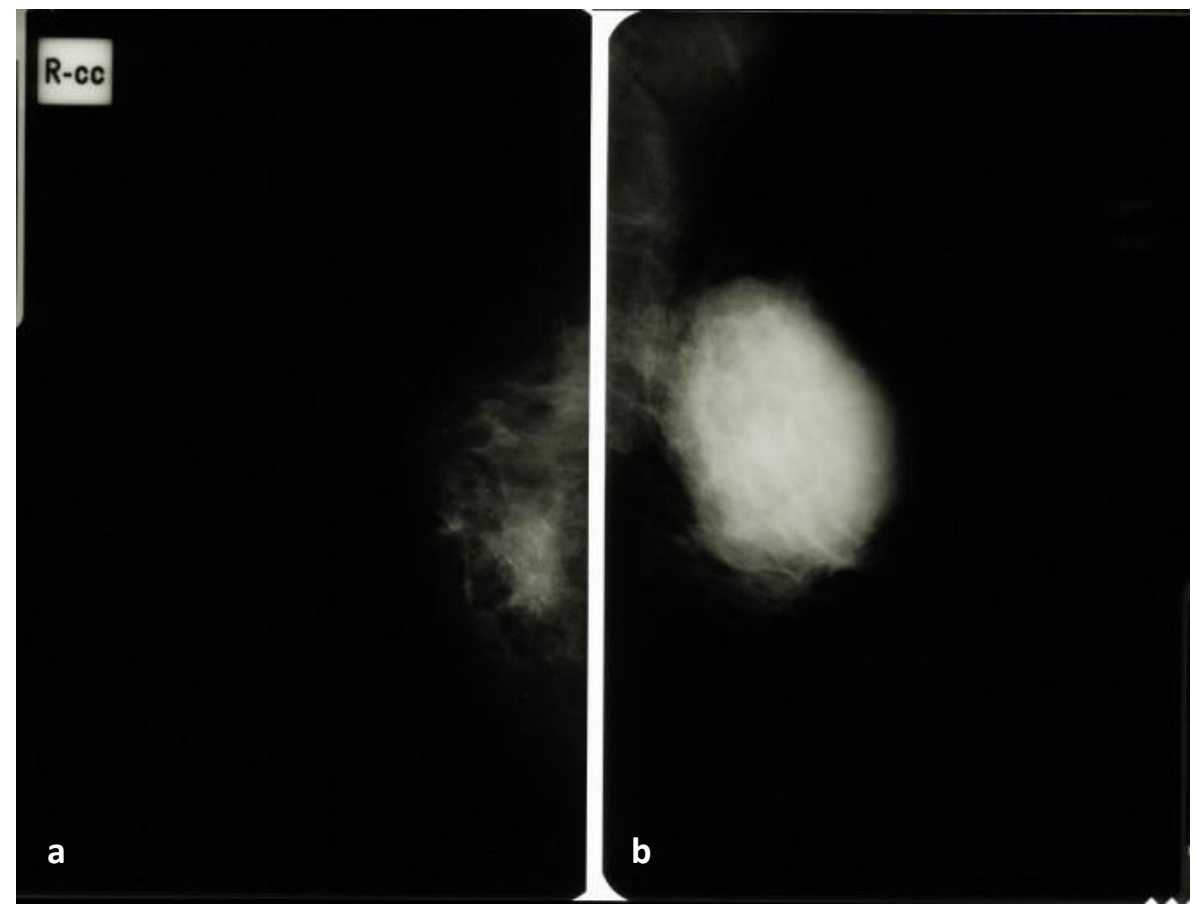

Fig. 1. Bilateral mammogram showing a $40 \mathrm{~mm}$ mass in the right breast, behind the nipple, with multiple microcalcifications (a) and a $90 / 75 \mathrm{~mm}$ mass in the left breast (b).

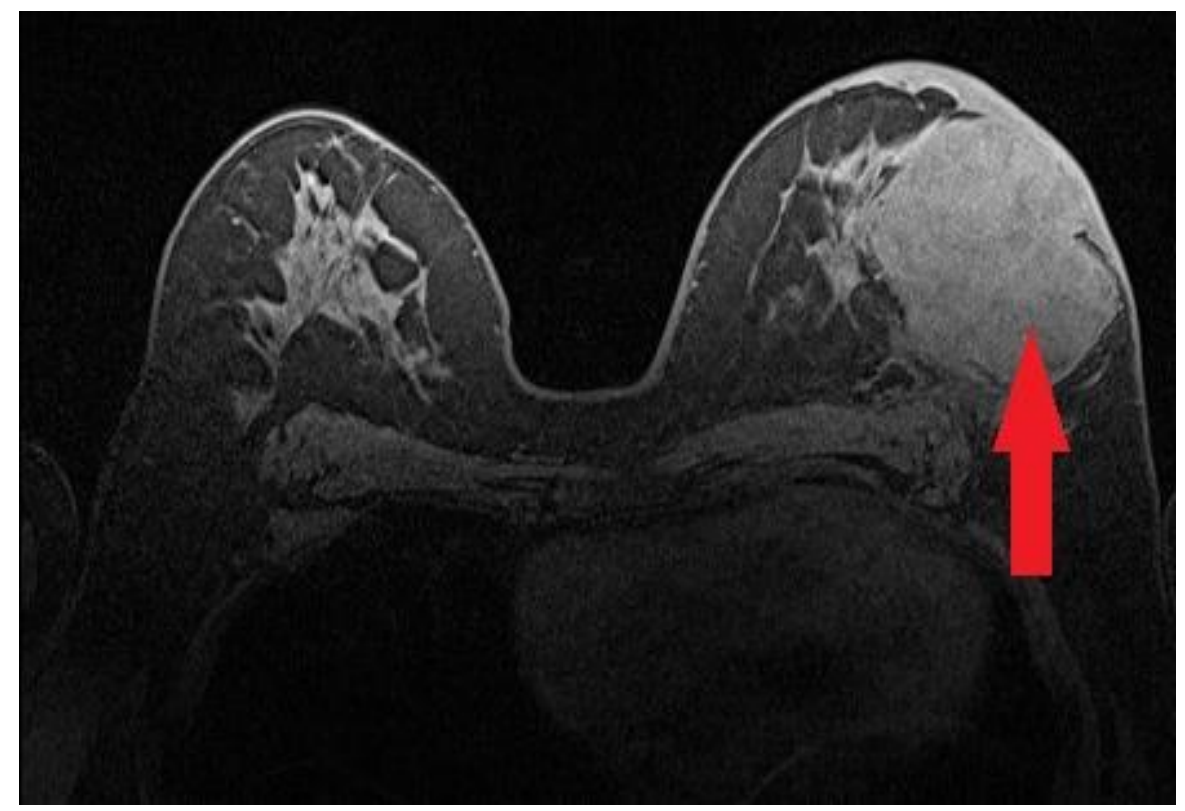

Fig. 2. Bilateral mammogram showing a mass with $90 / 75 \mathrm{~mm}$ on the left breast and a right mass behind the nipple with multiple microcalcifications, measuring $40 \mathrm{~mm}$. 
The brain, thoracic, abdominal and pelvic CT scan showed no metastasis. The final diagnosis was of left breast invasive carcinoma NST, luminal $\mathrm{B}, \mathrm{CT}_{4 \mathrm{~d}} \mathrm{~N}_{0} \mathrm{M}_{0}$, stage IIIB and right breast invasive carcinoma NST, luminal $\mathrm{B}, \mathrm{CT}_{3} \mathrm{~N}_{0} \mathrm{M}_{0}$, stage IIB.

The multidisciplinary team decided to proceed with neoadjuvant sequential chemotherapy based on 4 cycles of anthracyclines and 4 cycles of taxanes, which achieved partial response. A recommendation for genetic testing had been made, but the patient postponed it until 2017.

Systemic treatment was followed by bilateral modified radical mastectomy and laparoscopic bilateral adnexectomy (in order to avoid goserelin in adjuvant setting). Intraoperatively, bilateral ovarian tumors and miliary lesions of the peritoneum were objectified. Immunohistochemical test for these lesions revealed WT1 and PAX8 positivity, suggesting the ovarian origin and infirming a possible breast cancer metastasis. The pathology report concluded with the diagnosis of left breast tumor - $\mathrm{ypT}_{0} \mathrm{~N}_{0}(0 / 16)$ and right breast tumor - ypT $\mathrm{N}_{1}(2 / 15)$ and high grade serous ovarian carcinoma stage IIIB ( $y p T_{0} \mathrm{~N}_{1 a} \mathrm{M}_{1} \mathrm{~L}_{0} \mathrm{~V}_{0} \mathrm{R}_{0}$ ).

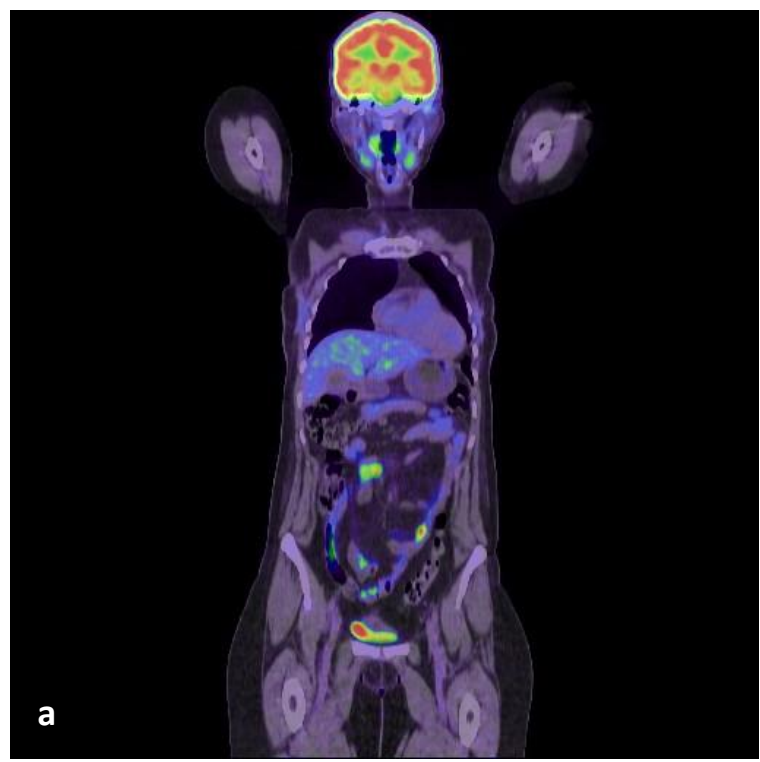

One month after surgery, the CT scan showed no residual tumors or metastasis. She received adjuvant external radiotherapy on both hemithoracic walls to a total dose of 50 Gy/ 25 fractions.

Because the surgery for ovarian cancer was bilateral adnexectomy alone, the first intention was to complete the procedure with hysterectomy and lymphadenectomy. According to the surgical report, R0 was impossible to achieve at that time due to peritoneal extension. Therefore, neoadjuvant chemotherapy based on platinum and taxanes was initiated ( 9 cycles) in parallel with adjuvant endocrine therapy (Letrozole) for breast cancer.

Imagistic evaluation at 3 months was performed in order to assess the best time for curative surgery with multiple resections performed in order to obtain complete response during the systemic treatment.

The PET-CT 6 months after the first surgery reported an active area (SUVmax $=7.2$ ) at the level of a mesogastric intestinal loop, one the left lower quadrant of the abdomen (SUVmax=9.7), with a reticular infiltration of the peritoneum in the left upper quadrant and the mesentery (Figure 3).

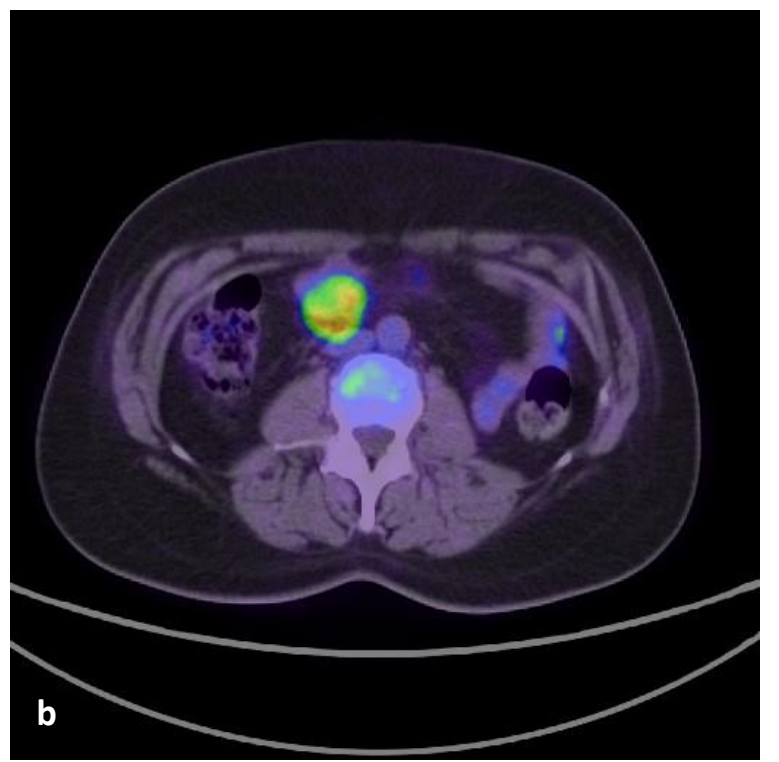

Fig. 3. FDG-PET-CT: (a) coronal section, active areas on the intestinal ansae (max SUV=9.7); (b) axial section, active area on the lower quadrant (max SUV=9.7). 
Genetic tests were performed from a blood sample in October 2017 and a BRCA1 germline mutation was identified (Cys61Gly). This variant is a common cause for breast and ovarian cancer in individuals of Eastern European ancestry. No other family members were tested. Chemotherapy was stopped in November 2017 after three laparoscopic exploratory interventions in order to assess the possibility to obtain clear surgical margins (at every 3 cycles) but, unfortunately, the disease showed transthoracic extension. Observation and close follow-up were considered the best option. One year after surgery the patient presented with an altered general health status, bradylalia, dysarthria and left hemiparesis.

Brain CT showed a left cerebral peduncle posterior pontin lesion $(23 / 16 \mathrm{~mm})$ with perilesional edema, most likely of breast origin in the context of normal ovarian tumoral marker CA-125 values $(12.1 \mathrm{U} / \mathrm{ml})$ and the known affinity for brain tissue of the breast cancer (Figure 4).

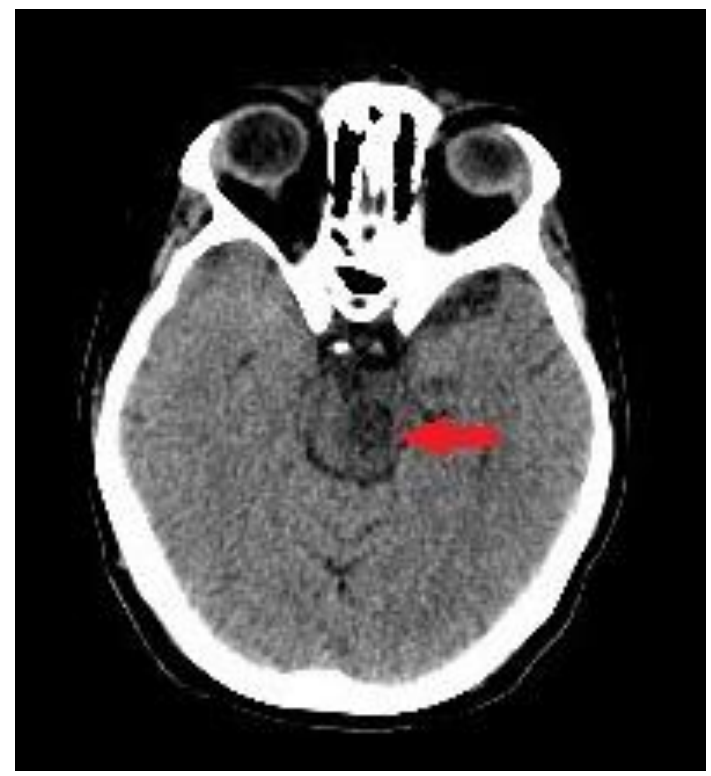

Fig. 4. Brain $\mathrm{CT}$, axial section, brainstem lesion measuring $20 \mathrm{~mm}$ (red arrow).

Palliative whole-brain radiotherapy (WBRT) was performed to a total dose of 30 Gy/10 fractions. At the end of the WBRT, the patient was still bradylalic with left hemiparesis. Through an early access programme, a PARP inhibitor (olaparib 150 $\mathrm{mg}$ BID) was initiated. A partial response by
RECIST 1.1 criteria (60\% reduction) was obtained after 3 months (Figure 5).

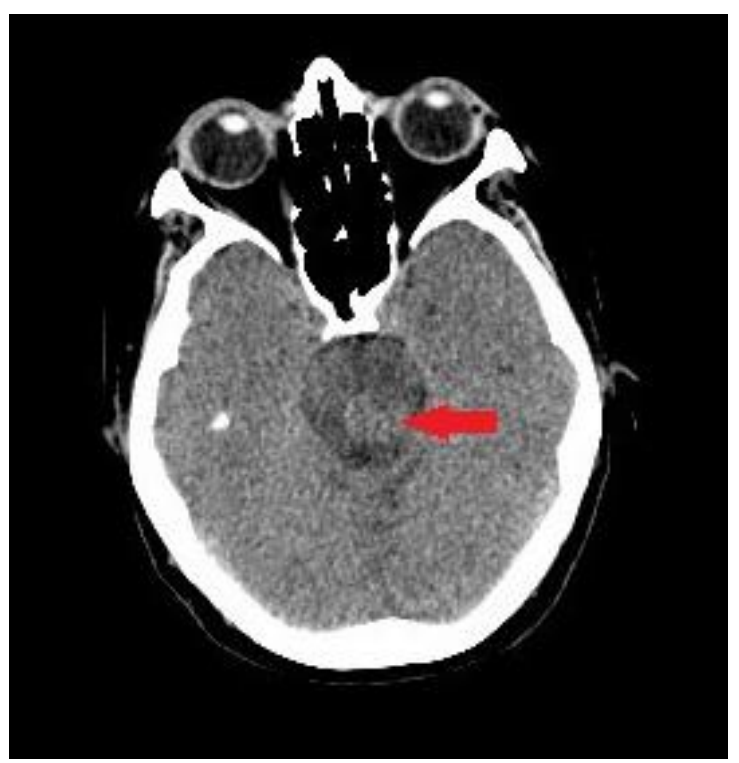

Fig. 5. Brain CT, axial section, brainstem lesion measuring $14 \mathrm{~mm}$ (red arrow).

After 6 months the patient presented with bradylalia, balance disorders and hemiplegia of the right lower limb, CT scan showing a lesion of $23 \mathrm{~mm}$ (Figure 6).

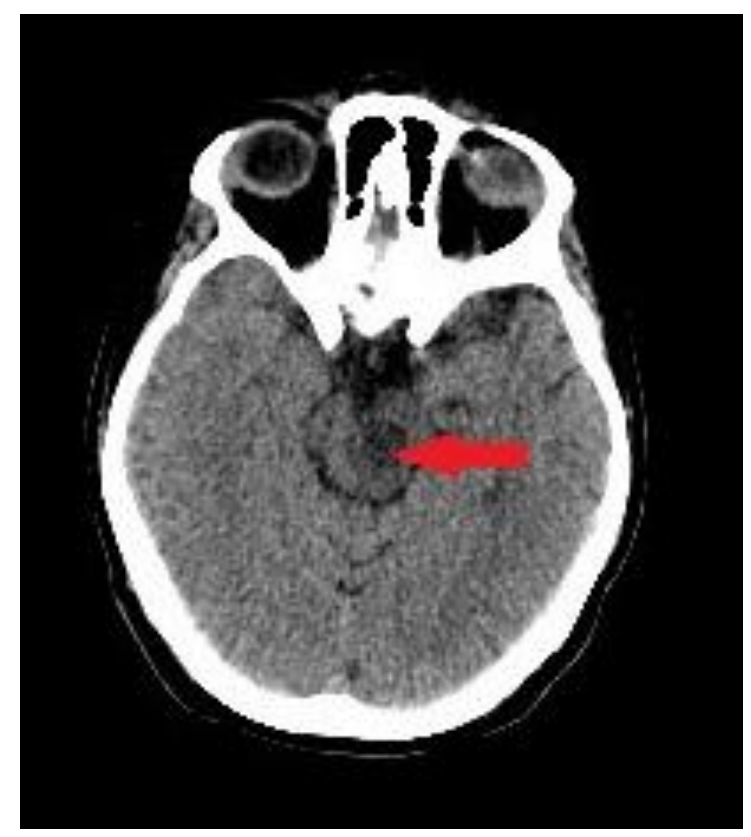

Fig. 6. Brain $\mathrm{CT}$, axial section, brainstem lesion measuring $23 \mathrm{~mm}$ (red arrow).

Second line chemotherapy with taxanes and bevacizumab obtained a complete 
radiologic response (Figure 7) and also clinical benefit after 4 cycles (Figure 8 ).

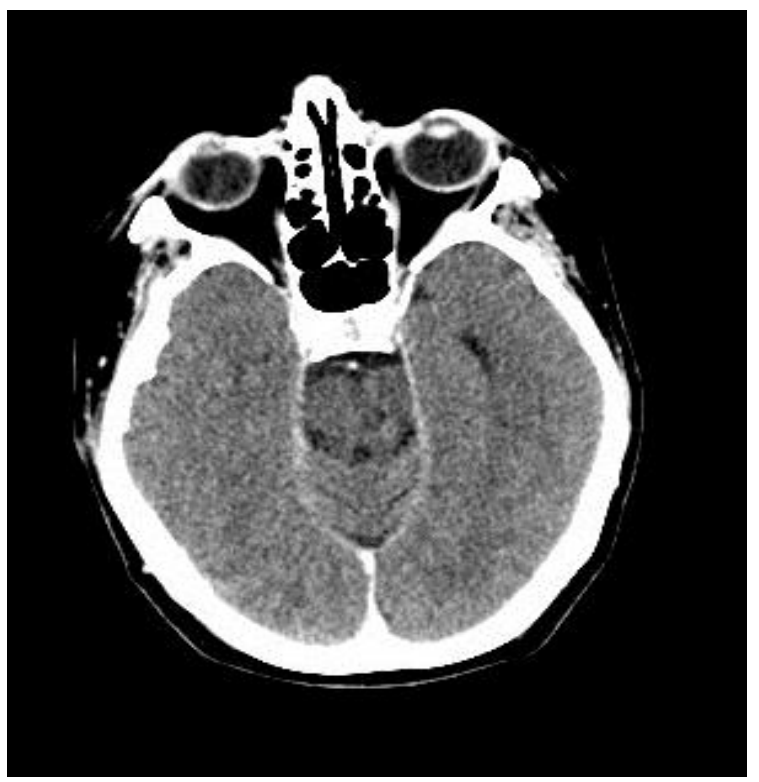

Fig. 7. Brain CT, axial section, no evidence of the brainstem lesion.

Regimen-response correlation

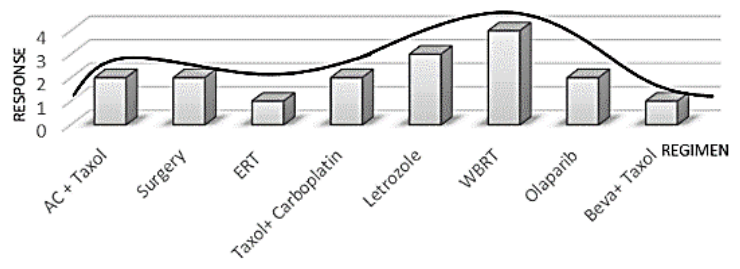

Fig. 8. Scheme of regimen and response correlation. 1 - CR (complete response), 2 - PR (partial response), 3 - SD (stable disease), 4 - PD (progression disease).

\section{Discussions}

The BRCA1 and BRCA2 genes are inherited in a dominant manner, and code for corresponding proteins with a protective role against tumors in organs like breast and ovaries [4].

BRCA germline mutations are responsible for a part of the familial breast and ovarian cancers. However, while many cases of synchronous or metachronous breast and ovarian cancers show a BRCA mutation, $70 \%$ of the cases might be caused by mutations in other genes [4]. The incidence of these tumors is reduced and post treatment survival is improved when gene mutations are detected early and risk-reducing treatment is administrated [4].

For a germline BRCA1-mutation carrier, the microenviroment tissue could play a significant role for breast cancer development by creating a pro-tumorigenic niche [5]. Only $10 \%$ of the ovarian cancers are caused by a genetic alteration. BRCA1 and BRCA2 mutations are present in up to $20 \%$ of the high grade serous ovarian cancer with an increased sensitivity under platinum regimens. High grade carcinoma is the most common subtype, with an incidence of $70 \%$ [6].

Synchronous bilateral breast cancer accounts for less than $2 \%$ of breast cancer cases. Five percent of all women diagnosed with bilateral breast carcinoma are thought to be BRCA mutation carriers [7].

Patients with bilateral breast cancer who present with synchronous malignancies have a greater risk for distant metastasis in comparison with women diagnosed with unilateral breast cancer and $6.4 \%$ have at least stage III breast cancer. Median time to distant metastasis was 63 months compared with 96 months for metachronous tumors [8]. The 8-years overall survival for synchronous disease was $78.7 \%$ versus $85.8 \%$ for the unilateral tumors [7].

Recurrence and death rates at 2 and 5 years are not different between patients with or without BRCA1 germline mutations, neither are the disease-free survival and overall survival [9]. BRCA germline mutation carriers bear an excessive risk for ovarian cancer (16.5-63\%), mostly higher-grade serous carcinomas [9].

The group of patients with a pathogenic BRCA variant presents an improved response to platinum chemotherapy, longer PFS and OS [10]. For our patient, genetic testing might have allowed diagnosis of the ovarian cancer at an earlier stage than discovered (FIGO IIIC), making a R0 resection easier to achieve.

BRCA1-associated breast cancers have a predilection for central nervous system metastasis [11].

Brain metastasis were observed in $3 \%$ of patients with BRCA mutations and only $1 \%$ of 
all ovarian cancer patients developed brain lesions [12]. This group of patients has a shorter interval to brain progression [11].

Olaparib is an oral PARP inhibitor which showed efficiency in BRCA-deficient advanced ovarian and breast cancer [13]. Recently, it has been approved by the FDA as monotherapy for BRCA mutated, HER2 negative metastatic breast cancer, after chemotherapy, based on significantly longer median progression-free survival (PFS) compared with standard chemotherapy (7 vs 4.2 months) [14]. Most adverse events with olaparib were grade 1 or 2, predominantly represented by nausea; main grade 3 or higher side effect was anemia [15].

According to the result of OlympiAD trial, $25.9 \%$ of the patients who received Olaparib were free of progression or death at 12 months, in comparison with $15 \%$ of the standard treatment group [15]. Unfortunately, our patient's condition progressed 6 months of PARP-inhibitor.

In addition to olaparib, other PARPinhibitors have been developed. Niraparib, rucaparib and veliparib are currently studied in BRCA-mutated breast and ovarian cancer [11].

In HER2-negative metastatic breast cancer patients with life-threatening disease or highly symptomatic, the addition of

\section{References}

1. $\mathrm{Yu}$ D, Hung MC. Breast cancer chemosensitivity, $1^{\text {st }}$ edition. New York: Springer-Verlag, 2007.

2. Dilawari A, Cangiarella J, Smith J, Huang A, Downey A, Muggia, F. Co-existence of breast and ovarian cancers in BRCA germ-line mutation carriers. Ecancermedicalscience 2008; 2:109.

3. Szarszewska M, Markowska A, Jach R et al. Significance of BRCA1 expression in breast and ovarian cancer patients with brain metastasis - A multicentre study. Adv Med Sci 2019; 64(2):235-240.

4. Asphaug L, Melberg $\mathrm{HO}$. The costeffectiveness of multigene panel testing for hereditary breast and ovarian cancer in Norway. MDM Policy Pract 2019; 4(1):2381468318821103.

5. Li CM, Oren Y, Regev A, Brugge JS. Contribution of mutant microenvironment to
Bevacizumab to a taxane-based regimen of chemotherapy brought significant improvement in PFS compared with chemotherapy alone [16, 17].

While it was not possible to perform a cerebral biopsy, we assumed that the brainstem lesion was due to the breast cancer. Having a BRCA-1 mutated gene, the use of Olaparib represented a better option compared to endocrine therapy.

The drug had been approved in Romania in February 2019 and the patient had access to it in June 2018.

\section{Conclusion}

The presence of synchronous breast and ovarian cancer at an older age justifies genetic testing. The role of genetic counselling and the availability of a drug like olaparib can increase survival and quality of life.

\section{Consent}

Written informed consent was obtained from the patient for publication of this case report.

\section{Competing interests}

The authors declare that they have no competing interests. hereditary cancer: Single-cell gene expression profiling of a genetically engineered mouse model of human hereditary BRCA1-related breast cancer [abstract]. In: Proceedings of the AACR Special Conference: Advances in Modeling Cancer in Mice: Technology, Biology, and Beyond; 2017 Sep 24-27; Orlando, Florida. Philadelphia (PA): AACR; Cancer Res 2018; 78(10 Suppl):Abstract nr PR06.

6. Manchana $T$, Lertkhachonsuk $R$, Teerapakpinyo C. Somatic BRCA mutation in high grade epithelial ovarian cancer patients. Asian Pac J Cancer Biol 2019, 3(4):99-103.

7. Heron DE, Komarnicky LT, Hyslop T, Schwartz GF, Mansfield CM. Bilateral breast carcinoma. Cancer 2000; 88(12):2739-2750.

8. Verhoog IC, Brekelmans CTM, Seynaeve C, et al. Survival and tumour characteristics of breast-cancer patients with germline mutations of BRCA1. Lancet 1998; 351(9099):316-321. 
9. Petrucelli N, Daly M B, Pal T. BRCA1- and BRCA2-associated hereditary breast and ovarian cancer. In: Adam MP, Ardinger $\mathrm{HH}$, Pagon RA, Wallace SE, Bean LJH, Stephens K, Amemiya A, editors. SourceGeneReviews ${ }^{\circledR}$ [Internet]. Seattle (WA): University of Washington, Seattle; 1993-2019. [https://www.ncbi.nlm.nih.gov/pubmed/2030142 5 available at 09.17.2019].

10. Ratner E, Bala M, Louie-Gao M, Aydin E, Hazard S, Brastianos PK. Increased risk of brain metastases in ovarian cancer patients with BRCA mutations. Gynecol Oncol 2019 153(3):568-573.

11. McCann KE, Hurvitz SA. Advances in the use of PARP inhibitor therapy for breast cancer. Drugs Context 2018; 7:212540. DOI: 10.7573/dic.212540.

12. Zavitsanos PJ, Wazer DE, Hepel JT, Wang Y, Singh K, Leonard KL. BRCA1 mutations associated with increased risk of brain metastases in breast cancer: a 1:2 matchedpair analysis. Am J Clin Oncol 2018; 41(12):1252-1256.
13. Tutt A, Robson M, Garber JE, et al. Oral poly (ADP-ribose) polymerase inhibitor olaparib in patients with BRCA1 or BRCA2 mutations and advanced breast cancer: a proof-of-concept trial. Lancet 2010; 376(9737):235-244.

14. Hartman M, Czene K, Reilly M, et al. Genetic implications of bilateral breast cancer: a population based cohort study. Lancet Oncol 2005; 6(6):377-382.

15. Robson M, Im SA, Senkus E, et al. Olaparib for metastatic breast cancer in patients with a germline BRCA mutation. N Engl J Med 2017; 377:523-533.

16. Valachis A, Polyzos NP, Patsopoulos NA, Georgoulias V, Mavroudis D, Mauri D. Bevacizumab in metastatic breast cancer: a meta-analysis of randomized controlled trials. Breast Cancer Res Treat 2010; 122(1):1-7.

17. NCCN Clinical Practice Guidelines in Oncology (NCCN Guidelines $\AA$ ). Breast Cancer. Screening and Diagnosis. Version 1.2019 [https://www2.tri-

kobe.org/nccn/guideline/breast/english/breastscreening.pdf available at 09.18.2019] 